University of South Carolina

Scholar Commons

4-1984

Error-bounds for Gaussian Quadrature and Weighted-L1

Polynomial Approximation

Ronald A. DeVore

University of South Carolina - Columbia, ronald.a.devore@gmail.com

L R. Scott

Follow this and additional works at: https://scholarcommons.sc.edu/math_facpub

Part of the Mathematics Commons

\title{
Publication Info
}

Siam Journal on Mathematical Analysis, Volume 21, Issue 2, 1984, pages 400-412.

(c) 1984 by Society for Industrial and Applied Mathematics

This Article is brought to you by the Mathematics, Department of at Scholar Commons. It has been accepted for inclusion in Faculty Publications by an authorized administrator of Scholar Commons. For more information, please contact digres@mailbox.sc.edu. 


\title{
ERROR BOUNDS FOR GAUSSIAN QUADRATURE AND WEIGHTED-L ${ }^{1}$ POLYNOMIAL APPROXIMATION*
}

\author{
RONALD A. DEVORE $\dagger$ AND L. RIDGWAY SCOTT $\ddagger$
}

\begin{abstract}
Error bounds for Gaussian quadrature are given in terms of the number of quadrature points and smoothness properties of the function whose integral is being approximated. An intermediate step involves a weighted- $L^{1}$ polynomial approximation problem which is treated in a more general context than that specifically required to bound the Gaussian quadrature error.
\end{abstract}

AMS(MOS) subject classifications. 65D30, 41 A55, 41A10

1. Introduction. The purpose of this note is to establish error bounds for Gaussian quadrature that reflect the fact that the accuracy is not degraded by certain singularities in the function to be integrated if they occur at the boundaries of the interval of integration rather than in the interior. It is well known that polynomial approximation can achieve greater accuracy at the boundary than in the interior (cf. Timan [5, p. 262]). The basic result to be derived says that the error in $N$-point Gaussian quadrature approximating the integral of $f$ on $[-1,1]$ is bounded by

$$
C_{s} N^{-s} \int_{-1}^{1}\left|f^{(s)}(x)\right|\left(1-x^{2}\right)^{s / 2} d x
$$

for all integers $s \leqq 2 N$ such that the above integral makes sense. Here, $C_{s}$ is a constant independent of $N$ and $f$. The main point of course is that $f$ may have certain singularities at $x= \pm 1$ such that the above integral is finite for some value of $s$, whereas it would not be finite if the same singularities occurred for $-1<x<1$. If $f$ has no singularities in the interval $[-1,1]$, then an error bound such as $(1.1)$ is not of great interest, as one would expect an exponential rate of convergence in this case (cf. Davis and Rabinowitz [1, p. 239]).

Theorem 3 below gives a result slightly sharper than (1.1) which allows one to interpret (1.1) as being valid for nonintegral $s$ via an interpolation argument. This interpolation argument can be used to determine, for example, the rate of convergence with respect to $N$ for a function with a power law singularity at the boundary (see Remark 4 in $\S 4$ ). Thus bounds of the type (1.1) appear to be the right way to predict the accuracy of Gaussian quadrature when applied to general classes of singular functions. However, our main motivation for proving (1.1) came from the so-called discrete ordinates method for the transport equation [3]. To bound the error in this method, one must consider the error in Gaussian quadrature applied to a one-parameter family of (singular) functions $f_{y}(x)$, where the strength of the singularity of $f$ at the boundary (in $x$ ) varies with $y$. Moreover, the singularities are not simple power law singularities, so the estimate (1.1) appeared essential to handle this problem.

${ }^{*}$ Received by the editors August 18,1981, and in revised form July 15, 1983. This research was sponsored by the U.S. Army under grant DAAG29-80-C-0041. It is based upon work supported by the National Science Foundation under grants MCS-81-01661 and MCS-81-01631.

† Department of Mathematics and Statistics, University of South Carolina, Columbia, South Carolina 29208.

‡ Department of Mathematics, University of Michigan, Ann Arbor, Michigan 48109. 
Bounds of the type (1.1) can also be given for composite rules using $N$ points on a mesh that is quadratically graded near \pm 1 , provided the basic rule has order of accuracy $s$ (see Remark 5 in $\S 4$ ). The techniques of proof for the case of composite rules are more elementary than those in the single-interval case. We include this simply for comparison and completeness.

In the following, we shall use interchangeably the words "bound" and "estimate," i.e. we shall refer occasionally to (1.1) as an "error estimate" even though it is not an asymptotic estimate of the actual error, rather simply an "estimate from above."

Our technique of proof is in two parts. Firstly, we analyze a Peano kernel for the quadrature error. This has the effect of establishing the bound (1.1) for $s=1$ and reducing further estimates to a weighted $L^{1}$ approximation problem for polynomials. This part of the analysis is presented in $\S 2$. Secondly, we consider weighted- $L^{1}$ approximation by polynomials and prove estimates for the error. This is done in $\S 3$.

Finally, the estimates of $\S \S 2$ and 3 are combined in $\S 4$ to give error bounds for Gaussian quadrature of the type (1.1) for all $s \geqq 1$. The calculations in $\S 2$ are done specifically for Gaussian quadrature, although they clearly extend to more general quadrature approximations based on orthogonal polynomials for weighted integrals.

The weighted $L^{1}$ approximation estimates show that, for a rather general class of weights $w>0$ and sufficiently smooth $f$, there is a polynomial $P$ of degree at most $N$ so that

$$
\int_{-1}^{1}|f(x)-P(x)| w(x) d x \leqq C_{s} N^{-s} \int_{-1}^{1}\left|f^{(s)}(x)\right|(1-x)^{s / 2} w(x) d x
$$

for any $s$ and $N$ such that $N+1 \geqq s \geqq 1$. In the case $w \equiv 1$ and $s=1,(1.2)$ has been established by N. X. Ky [2].

2. Error estimates for Gaussian quadrature. Consider Gaussian quadrature approximation of the form

$$
\int_{-1}^{1} f(x) d x \sim \sum_{j=1}^{N} \omega_{j} f\left(x_{j}\right)=: I_{N}(f)
$$

$\left(\left\{x_{j}\right\}\right.$ are the zeros of the Legendre polynomials and $\left\{\omega_{j}\right\}$ are the integrals of the associated Lagrange interpolation polynomials, cf. G. Szegö [4]). The ordering $-1<x_{1}<\cdots<x_{N}<1$ will be assumed, and we introduce $x_{0}:=-1$ and $x_{N+1}:=1$. We wish to establish estimates of the error

$$
e_{N}(f):=\int_{-1}^{1} f(x) d x-I_{N}(f)
$$

in terms of $N$ and properties of $f$.

Assume $N \geqq 2$ (our final estimate in Theorem 1 will hold for $N=1$ as well, as may be easily checked). The Peano kernel theorem allows us to write

$$
e_{N}(f)=\int_{-1}^{1} K(t) f^{\prime}(t) d t
$$

at least for smooth $f$, where $K(t)=e_{N}\left(H_{t}\right),|t| \leqq 1$, (cf. (3.3) below) and $H_{t}$ is the Heaviside function

$$
H_{t}(x):= \begin{cases}0, & x<t \\ 1, & x \geqq t\end{cases}
$$


It follows that

$$
K(t)=1-t-\sum_{x_{j}>t} \omega_{j}=\sum_{x_{j}<t} \omega_{j}-t-1
$$

The Chebyshev-Markov-Stieltjes inequality (cf. G. Szegö [4, p. 50]) implies that

$$
1+x_{j} \leqq \sum_{i=1}^{j} \omega_{i} \leqq 1+x_{j+1}, \quad j=1, \cdots, N .
$$

Therefore, for $j=1, \cdots, N$,

$$
x_{j-1}-x_{j} \leqq K\left(x_{j}-\right) \leqq 0 \leqq K\left(x_{j}+\right) \leqq x_{j+1}-x_{j} .
$$

Since $K$ vanishes in each interval $\left[x_{j-1}, x_{j}\right]$ and its slope is one almost everywhere,

$$
\max \left\{|K(x)|: x \in\left[x_{j-1}, x_{j}\right]\right\} \leqq x_{j}-x_{j-1},
$$

for $j=1, \cdots, N+1$. To bound $x_{j}-x_{j-1}$, recall that (cf. G. Szegö [4, p. 122])

$$
x_{j}=-\cos \theta_{j} \quad \text { where } \frac{(2 N+1) \theta_{j}}{\pi} \in[2 j-1,2 j],
$$

for $j=1, \cdots, N$. Thus

$$
\begin{aligned}
x_{j}-x_{j-1}=\cos \theta_{j-1}-\cos \theta_{j} & =\int_{\theta_{j-1}}^{\theta_{j}} \sin \theta d \theta \\
& \leqq\left(\theta_{j}-\theta_{j-1}\right) \max \left\{\sin \theta: \theta \in\left[\theta_{j-1}, \theta_{j}\right]\right\} \\
& \leqq\left(\frac{3 \pi}{2 N}\right) \max \left\{\sin \theta: \theta \in\left[\theta_{j-1}, \theta_{j}\right]\right\} .
\end{aligned}
$$

To bound the latter term, observe that, since $(\sin \theta) / \theta$ is decreasing on $[0, \pi],(2.4)$ implies

$$
\sin \theta \leqq\left(\frac{\theta}{\theta_{j-1}}\right) \sin \theta_{j-1} \leqq\left(\frac{\theta_{j}}{\theta_{j-1}}\right) \sin \theta_{j-1} \leqq 4 \sin \theta_{j-1}
$$

for $\theta \in\left[\theta_{j-1}, \theta_{j}\right]$ and $j=2, \cdots, N$. From the symmetry properties of the $\theta_{j}$ 's (cf. [4]), it then follows that

$$
\sin \theta \leqq 4 \sin \theta_{j}, \quad \theta \in\left[\theta_{j-1}, \theta_{j}\right], \quad j=2, \cdots, N
$$

as well. Thus we have

$$
\max \left\{\sin \theta: \theta \in\left[\theta_{j-1}, \theta_{j}\right]\right\} \leqq 4 \min \left\{\sin \theta: \theta \in\left[\theta_{j-1}, \theta_{j}\right]\right\}
$$

for $j=2, \cdots, N$. Thus we find, for $j=2, \cdots, N$,

$$
\begin{aligned}
x_{j}-x_{j-1} & \leqq 6 \pi \min \left\{\sin \theta: \theta \in\left[\theta_{j-1}, \theta_{j}\right]\right\} / N \\
& =6 \pi \min \left\{\sqrt{1-\cos ^{2} \theta}: \theta \in\left[\theta_{j-1}, \theta_{j}\right]\right\} / N \\
& =6 \pi \min \left\{\sqrt{1-x^{2}}: x \in\left[x_{j-1}, x_{j}\right]\right\} / N .
\end{aligned}
$$

Therefore, we have

$$
|K(x)| \leqq \frac{6 \pi \sqrt{1-x^{2}}}{N}
$$


at least for $x$ between $x_{1}$ and $x_{N}$. For $x \in\left[-1, x_{1}\right]$

$$
\begin{aligned}
|K(x)| \leqq 1+x & =\frac{\sqrt{1-x^{2}} \sqrt{1+x}}{\sqrt{1-x}} \leqq \sqrt{1-x^{2}} \sqrt{1+x_{1}} \\
& =\sqrt{1-x^{2}} \sqrt{1-\cos \theta_{1}} \leqq \sqrt{1-x^{2}} \frac{\theta_{1}}{\sqrt{2}} \leqq \frac{\sqrt{1-x^{2}} \pi}{\sqrt{2} N}
\end{aligned}
$$

A corresponding estimate holds by symmetry for $x \in\left[x_{N}, 1\right]$, so (2.6) is valid for all $x \in[-1,1]$. Thus we have proved that

$$
\left|e_{n}(f)\right| \leqq 6 \pi N^{-1} \int_{-1}^{1}\left|f^{\prime}(x)\right| \sqrt{1-x^{2}} d x .
$$

Since (2.4) implies that

$$
\frac{1}{N} \leqq \sin \left(\frac{\pi}{2 N+1}\right) \leqq \sqrt{1-x^{2}}
$$

for $x \in\left[x_{1}, x_{N}\right]$ and $N \geqq 2,(2.6)$ implies further that

$$
|K(x)| \leqq 6 \pi\left(1-x^{2}\right)
$$

for $x \in\left[x_{1}, x_{N}\right]$. But for $x \in\left[-1, x_{1}\right] \cup\left[x_{N}, 1\right],|K(x)|=1-|x|$. Thus (2.7) holds for all $x \in[-1,1]$, giving the following estimate:

$$
\left|e_{N}(f)\right| \leqq 6 \pi \int_{-1}^{1}\left|f^{\prime}(x)\right| \min \left\{\frac{\sqrt{1-x^{2}}}{N}, 1-x^{2}\right\} d x
$$

These estimates hold for all $f \in L^{1}([-1,1])$ whose weak derivatives are integrable with respect to the weight $1-x^{2}$, as can be seen by approximating $f$ via smooth functions (cf. the definition of the space $Y_{w}^{s}$ given later in $\S 3$ ). Summarizing the above, we have the following:

THEOREM 1. Let $e_{N}(f)$ denote the error in $N$-point Gaussian quadrature applied to $f \in L^{1}([-1,1])$ (see (2.1)-(2.2) for definitions). If the weak derivative, $f^{\prime}$, of $f$ is integrable with respect to the weight $1-x^{2}$, then

$$
\left|e_{N}(f)\right| \leqq 6 \pi \int_{-1}^{1}\left|f^{\prime}(x)\right| \min \left\{\frac{\sqrt{1-x^{2}}}{N}, 1-x^{2}\right\} d x
$$

If $f^{\prime}$ is integrable with respect to $\sqrt{1-x^{2}}$, then

$$
\left|e_{N}(f)\right| \leqq \frac{6 \pi}{N} \int_{-1}^{1}\left|f^{\prime}(x)\right| \sqrt{1-x^{2}} d x
$$

Estimates involving higher derivatives of $f$ can also be derived by estimating Peano kernels. For example, one may write, for any $1 \leqq k \leqq 2 N$,

$$
e_{N}(f)=\int_{-1}^{1} K_{k}(x) f^{(k)}(x) d x
$$


( $K_{1}=K$ in the previous notation). $K_{k}$ is a $C^{(k-2)}$ piecewise ( $k$ th degree) polynomial (with knots $\left\{x_{i}\right\}$ ) satisfying $0=K_{k}( \pm 1)=\cdots=K_{k}^{(k-1)}( \pm 1)$ and $K_{k}^{(k)}=(-1)^{k}$ between knots. Moreover, $K_{k}^{(k-1)}\left(x_{j}+\right)-K_{k}^{(k-1)}\left(x_{j}-\right)=(-1)^{k-1} \omega_{j}, j=1, \cdots, N$. Using these facts together with a special oscillation property of $K_{2}$, it can be shown that

$$
\left|K_{2}(x)\right| \leqq C \min \left\{\frac{1-x^{2}}{N^{2}},\left(1-x^{2}\right)^{2}\right\} .
$$

However, it becomes increasingly difficult to estimate the higher-degree kernels $K_{k}$, $k \geqq 3$. Thus the following approach proves more fruitful.

Observe that $e_{N}(f)=e_{N}(f-P)$ for any $P \in \mathscr{P}_{2 N-1}$, where $\mathscr{P}_{r}$ denotes the set of polynomials of degree not exceeding $r$. Therefore, Theorem 1 implies that

$$
\left|e_{N}(f)\right| \leqq \inf _{P \in \mathscr{P}_{2 N-2}} \int_{-1}^{1}\left|f^{\prime}-P\right|(x) w(x) d x,
$$

where $w$ is the weight function

$$
w(x)=6 \pi \min \left\{\frac{\sqrt{1-x^{2}}}{N}, 1-x^{2}\right\} .
$$

Thus, estimates of $e_{N}(f)$ are reduced to a weighted- $L^{1}$ approximation problem for polynomials. Such problems will be considered in the next section. The results of $\S 3$ on approximation will be combined in $\S 4$ with the results of this section to give higher order estimates of the form

$$
\left|e_{N}(f)\right| \leqq C_{s} \int_{-1}^{1}\left|f^{(s)}(x)\right| \min \left\{\left(\frac{\sqrt{1-x^{2}}}{N}\right)^{s},\left(1-x^{2}\right)^{s}\right\} d x
$$

for arbitrary positive integers $s$ and $N$ such that $N \geqq s / 2$.

3. Weighted- $L^{1}$ approximation. In this section, we shall prove a weighted- $L^{1}$ polynomial approximation result of the form

$$
\inf _{P \in \mathscr{P}_{N}} \int_{-1}^{1}|u-P|(x) w(x) d x \leqq C_{s, w} N^{-s} \int_{-1}^{1}\left|u^{(s)}(x)\right| w(x)\left(1-x^{2}\right)^{s / 2} d x
$$

where $\mathscr{P}_{N}$ denotes the set of polynomials of degree not exceeding $N, s$ is a positive integer, $C_{s, w}$ is a constant independent of $N$ and $u$, and $w$ is a positive, integrable weight function to be discussed in more detail subsequently. Rather than state our abstract conditions on $w$ and $u$ initially, we shall develop them in the course of deriving (3.1). However, suffice it to say that (3.1) will be proved for a class of weights including the Jacobi weights

$$
w(x)=(1+x)^{\alpha}(1-x)^{\beta}, \quad \alpha, \beta>-1 .
$$

As a first step, recall the Heaviside function $H_{t}(x)$ defined in (2.3). If $u$ is sufficiently smooth, we may write

$$
u(x)=P_{1}(x)+\frac{1}{(s-1) !} \int_{-1}^{1} H_{t}(x)(x-t)^{s-1} u^{(s)}(t) d t
$$

for all $x \in[-1,1]$, where $P_{1} \in \mathscr{P}_{s-1}$. Let $\lambda_{t} \in \mathscr{P}_{N-s+1}$ be an arbitrary family depending, say, piecewise continuously on $t$, and define

$$
P(x)=P_{1}(x)+\frac{1}{(s-1) !} \int_{-1}^{1} \lambda_{t}(x)(x-t)^{s-1} u^{(s)}(t) d t .
$$


Then $P \in \mathscr{P}_{N}$, and Hölder's inequality and Fubini's theorem imply that

$$
\int_{-1}^{1}|u-P|(x) w(x) d x \leqq \frac{1}{(s-1) !} \int_{-1}^{1}\left\{\int_{-1}^{1}\left|\lambda_{t}-H_{t}\right|(x)|x-t|^{s-1} w(x) d x\right\}\left|u^{(s)}(t)\right| d t .
$$

Thus to prove (3.1), it suffices to construct $\lambda_{t}$ in such a way that

$$
\int_{-1}^{1}\left|\lambda_{t}-H_{t}\right|(x)|x-t|^{s-1} w(x) d x \leqq C_{s} N^{-s}\left(1-t^{2}\right)^{s / 2} w(t) .
$$

For $t$ near \pm 1 , this is relatively easy to do under the following assumption:

(A1) . There is a constant $A_{1}$ such that

$$
\int_{t}^{1} w(x) d x \leqq A_{1}(1-t) w(t) \quad \text { for } 0 \leqq t \leqq 1
$$

and

(ii)

$$
\int_{-1}^{t} w(x) d x \leqq A_{1}(1+t) w(t) \quad \text { for }-1 \leqq t \leqq 0 .
$$

EXAMPLE 1. Assumption (A1) holds for the Jacobi weight $w(x)=$ $(1+x)^{\alpha}(1-x)^{\beta}$ provided $\alpha, \beta>-1$.

Proof. To see this, it suffices to verify, say, (i). Then

$$
\begin{aligned}
\int_{t}^{1} w(x) d x & =\int_{t}^{1}(1+x)^{\alpha}(1-x)^{\beta} d x \leqq 2^{\max \{\alpha, 0\}} \int_{t}^{1}(1-x)^{\beta} d x \\
& \leqq \frac{2^{\max \{\alpha, 0\}}(1-t)^{1+\beta}}{1+\beta} \leqq \frac{2^{|\alpha|}}{1+\beta}(1+t)^{\alpha}(1-t)^{1+\beta}=A_{1}(1-t) w(t) .
\end{aligned}
$$

Lemma. Suppose Assumption (A1) holds. Define $\lambda_{t}$ by

$$
\lambda_{t}(x):=\left\{\begin{array}{lll}
0 & \text { for all } x \in[-1,1] & \text { if } t>0 \\
1 & \text { for all } x \in[-1,1] & \text { if } t \leqq 0
\end{array}\right.
$$

Then

$$
\int_{-1}^{1}\left|\lambda_{t}-H_{t}\right|(x)|x-t|^{s-1} w(x) d x \leqq A_{1}\left(1-t^{2}\right)^{s} w(t)
$$

for all $t \in[-1,1]$.

Proof. Suppose $t>0$. From (2.3) and Assumption (A1), we have

$$
\begin{aligned}
\int_{-1}^{1}\left|\lambda_{t}-H_{t}\right|(x)|x-t|^{s-1} w(x) d x & =\int_{t}^{1}|x-t|^{s-1} w(x) d x \leqq(1-t)^{s-1} \int_{t}^{1} w(x) d x \\
& \leqq A_{1}(1-t)^{s} w(t) \leqq A_{1}\left(1-t^{2}\right)^{s} w(t) .
\end{aligned}
$$

The case $t \leqq 0$ is similar.

COROLLARY. Let $\kappa \geqq 1$ be arbitrary, and suppose (A1) holds. Then for any $N \geqq 1$ and $t \in[-1,1]$ such that $\sqrt{1-t^{2}} \leqq \kappa / N$, the choices for $\lambda_{t}$ given in the lemma satisfy the estimate (3.6) with $C_{s}=A_{1} \kappa^{s}$. 
In view of this corollary, it suffices to assume that $\sqrt{1-t^{2}} \geqq \kappa / N$, where $\kappa$ can be chosen later at our discretion. We shall subsequently construct, for certain values of $t, \lambda_{t}=\lambda_{t, r, N} \in \mathscr{P}_{N-2 r+1}$ such that

$$
\left|H_{t}-\lambda_{t}\right|(x) \leqq C_{r}\left(\frac{\sqrt{1-t^{2}}}{N}\right)^{2 r-1}|x-t|^{-2 r+1} \text { for } x \in[-1,1],
$$

where $r$ is any positive integer (to be chosen later depending on $w$ and $s$ ). Furthermore, $\lambda_{t}$ will be monotone, nondecreasing, with $\lambda_{t}(-1)=0$ and $\lambda_{r}(+1)=1$. Hence also

$$
\left|H_{t}-\lambda_{t}\right|(x) \leqq 1 \quad \text { for all } x \in[-1,1] \text {. }
$$

Assuming these properties of $\lambda_{t}$ for some $t$ for the moment, we proceed to prove (3.6) for such $t$. Let $\delta=\sqrt{1-t^{2}} / N$. Then

$$
\begin{aligned}
& \int_{-1}^{1}\left|H_{t}-\lambda_{t}\right||x-t|^{s-1} w(x) d x \\
& \quad \leqq \int_{\substack{|x-t| \leq \delta \\
|x| \leqq 1}}|x-t|^{s-1} w(x) d x+C_{r} \int_{\substack{|x-t| \geqq \delta \\
|x| \leqq 1}}|x-t|^{s-2 r} w(x) d x \delta^{2 r-1},
\end{aligned}
$$

where we used the bounds (3.8) and (3.7) on the first and second integrals, respectively. Thus the estimate (3.6) follows easily from the following two assumptions:

(A2) Let $s$ be a positive integer. Then there exist constants $A_{2}<\infty$ and $2 \leqq \gamma<\infty$ such that, for all $\delta>0$ and $t \in[-1,1]$ satisfying $1-t^{2} \geqq \gamma \delta$,

$$
\int_{|x| \leqq \delta} w(x+t)|x|^{s-1} d x \leqq A_{2} w(t) \delta^{s} .
$$

(A3) There exist $A_{3}<\infty, 2 \leqq \gamma<\infty$ and $0<k_{0}<\infty$ such that, for all $\delta>0$ and $t \in[-1,1]$ satisfying $1-t^{2} \geqq \gamma \delta$ and all $k \geqq k_{0}$,

$$
\int_{\substack{|x| \geqq \delta \\|t+x| \leqq 1}} w(x+t)|x|^{-k} d x \leqq A_{3} w(t) \delta^{-k+1} .
$$

In applying (A2) and (A3) to (3.9), note that, for $\delta=\sqrt{1-t^{2}} / N$, the condition $1-t^{2} \geqq \gamma \delta$ is equivalent to $\sqrt{1-t^{2}} \geqq \gamma / N$. This will therefore be satisfied if $\kappa \geqq \gamma$, a requirement we now impose on $\kappa$. Choosing $r$ such that $2 r-s=: k \geqq k_{0}$ thus proves (3.6) for $t$ such that (3.7)-(3.8) hold. Before proving the bounds (3.7) and (3.8), we show that (A2) and (A3) hold for Jacobi weights.

EXAMPLE 2. Assumption (A2) holds for the Jacobi weights $w(x)=(1+x)^{\alpha}(1-x)^{\beta}$ for all $\alpha, \beta \in R$.

Proof. Take $\gamma=3$. By a change of variables,

$$
\begin{aligned}
\delta^{-s} \int_{|x| \leqq \delta} \frac{w(x+t)}{w(t)}|x|^{s-1} d x & =\delta^{-s} \int_{|x| \leqq \delta}\left(1+\frac{x}{1+t}\right)^{\alpha}\left(1-\frac{x}{1-t}\right)^{\beta}|x|^{s-1} d x \\
& =\int_{|y| \leqq 1}\left(1+\left(\frac{\delta}{1+t}\right) y\right)^{\alpha}\left(1-\left(\frac{\delta}{1-t}\right) y\right)^{\beta}|y|^{s-1} d y
\end{aligned}
$$

But for $1-t^{2} \geqq 3 \delta, \delta /(1 \pm t) \in\left[0, \frac{2}{3}\right]$. Thus the integrand is bounded by $3^{\max \{|\alpha|,|\beta|\}}$. 
EXAMPLE 3. Assumption (A3) holds for the Jacobi weights $w(x)=(1+x)^{\alpha}(1-x)^{\beta}$ for all $\alpha, \beta>-1$.

Proof. Take $\gamma=2$ and $k_{0}:=2+\max \{\alpha, \beta, 0\}$. Then

$$
\begin{aligned}
& \delta^{k-1} \int_{\substack{|x| \geqq \delta \\
|x+t| \leqq 1}} \frac{w(x+t)}{w(t)}|x|^{-k} d x \\
& =\delta^{k-1}\left(\int_{\delta}^{1-t} \frac{w(x+t)}{w(t)} x^{-k} d x+\int_{\delta}^{1+t} \frac{w(t-x)}{w(t)} x^{-k} d x\right) \\
& =\delta^{k-1}\left(\int_{\delta}^{1-t}\left(1+\frac{x}{1+t}\right)^{\alpha}\left(1-\frac{x}{1-t}\right)^{\beta} x^{-k} d x\right. \\
& \left.\quad \quad+\int_{\delta}^{1+t}\left(1-\frac{x}{1+t}\right)^{\alpha}\left(1+\frac{x}{1-t}\right)^{\beta} x^{-k} d x\right) \\
& =: I_{-}(t, \delta, \alpha, \beta, k)+I_{+}(t, \delta, \alpha, \beta, k) .
\end{aligned}
$$

Since $I_{+}(t, \delta, \alpha, \beta, k)=I_{-}(-t, \delta, \beta, \alpha, k)$, it suffices to show that, for all $|t| \leqq \sqrt{1-\gamma \delta}$,

$$
I_{-}(t, \delta, \alpha, \beta, k) \leqq C \quad \text { provided } k \geqq 2+\max \{\alpha, 0\} .
$$

By a change of variables, note that

$$
I_{-}(t, \delta, \alpha, \beta, k)=\int_{1}^{(1-t) / \delta}\left(1+\left(\frac{\delta}{1+t}\right) y\right)^{\alpha}\left(1-\left(\frac{\delta}{1-t}\right) y\right)^{\beta} y^{-k} d y .
$$

Let $M:=(1-t) / \delta$. Thus, for $\alpha \geqq 0$,

$$
\begin{aligned}
I_{-} & \leqq \int_{1}^{M}(1+y)^{\alpha}\left(1-M^{-1} y\right)^{\beta} y^{-k} d y \leqq 2^{\alpha} \int_{1}^{M}\left(1-M^{-1} y\right)^{\beta} y^{\alpha-k} d y \\
& \leqq 2^{\alpha} \int_{1}^{M}\left(1-M^{-1} y\right)^{\beta} y^{-2} d y .
\end{aligned}
$$

In the first estimate above, we used the fact that, for $|t| \leqq 1$,

$$
(1+t) \geqq \frac{1}{2}(1-t)(1+t) \geqq \frac{1}{2} \gamma \delta=\delta .
$$

For $\alpha<0$, one has

$$
I_{-} \leqq \int_{1}^{M}\left(1-M^{-1} y\right)^{\beta} y^{-k} d y \leqq \int_{1}^{M}\left(1-M^{-1} y\right)^{\beta} y^{-2} d y .
$$

A simple calculation shows that, for $M \geqq 1$,

$$
\int_{1}^{M}\left(1-M^{-1} y\right)^{\beta} y^{-2} d y \leqq C_{\beta} \quad \text { for all } \beta>-1,
$$

completing the proof.

We now construct $\lambda_{t}$ satisfying (3.7)-(3.8). We shall do so by choosing

$$
\lambda_{t}(x):=\int_{-1}^{x} \delta_{t}(y) d y
$$

where $\delta_{t} \in \mathscr{P}_{N-2 r}$ approximates the Dirac $\delta$-function. In particular, $\delta_{t}$ will be nonnegative and will have integral on $[-1,1]$ equal to one. Thus $\lambda_{t}$ satisfies the claimed monotonicity property as well as having the required values at \pm 1 . Hence (3.8) will follow automatically. 
To construct $\delta_{t}$, first note that it suffices to take $\delta_{t}(x):=\frac{1}{2}$ for all $x \in[-1,1]$ for $N<4 r$ (recall that we restrict $t$ to satisfying $\sqrt{1-t^{2}} \geqq \gamma / N$ in (3.7)-(3.8)). For $N \geqq 4 r$, let $n$ be the greatest integer not exceeding $N / 2 r$. Note that $N / n \leqq 3 r$. Let $T_{n}$ denote the Chebyshev polynomial of degree $n$, and let $\left\{t_{i}: 1 \leqq i \leqq n\right\}$ be its zeros:

$$
T_{n}(x):=\cos \left(n\left(\cos ^{-1} x\right)\right), \quad t_{i}:=\cos \left(\frac{(i-1 / 2) \pi}{n}\right)=: \cos \theta_{i} \quad i=1, \cdots, n .
$$

We shall establish (3.7)-(3.8) for $t \in\left\{t_{i}: 1 \leqq i \leqq n\right\}$. Define

$$
\delta_{t_{i}}(x):=c_{i}\left[\frac{T_{n}(x)}{\left(x-t_{i}\right)}\right]^{2 r}
$$

where $c_{i}$ is chosen so that $\int_{-1}^{1} \delta_{t_{i}}(x) d x=1$. We now estimate the size of $c_{i}$. Observe that $\delta_{t_{n+1-i}}(x)=\delta_{t_{i}}(-x)$, so that $c_{n+1-i}=c_{i}$. Thus we may suppose that $i \leqq(n+1) / 2$. Also, we have $n \geqq 2$. Furthermore,

$$
\left|T_{n}(x)\right|=|\cos n \theta| \geqq \frac{1}{2} \text { for }|n \theta-i \pi| \leqq \frac{\pi}{3} .
$$

Thus, writing $x=\cos \theta$, we see that for $|n \theta-i \pi| \leqq \pi / 3$.

$$
\begin{aligned}
\left|\frac{T_{n}(x)}{\left(x-t_{i}\right)}\right| & \geqq \frac{1}{2}\left|\cos \theta-\cos \theta_{i}\right|^{-1} \geqq \frac{1}{2}\left|\cos \left(\frac{(i+1 / 3) \pi}{n}\right)-\cos \theta_{i}\right|^{-1} \\
& \geqq\left[\left(\frac{5 \pi}{3 n}\right) \max \left\{\sin \theta:\left(\frac{n \theta}{\pi}\right)-i \in\left[-\frac{1}{2}, \frac{1}{3}\right]\right\}\right]^{-1} \geqq\left[\left(\frac{5 \pi}{n}\right) \sin \theta_{i}\right]^{-1},
\end{aligned}
$$

because $\sin \theta / \theta$ is decreasing on $[0, \pi]$. The measure of the set $\{x=\cos \theta:|n \theta-i \pi| \leqq$ $\pi / 3\}$ is

$$
\cos \left(\frac{(i-1 / 3) \pi}{n}\right)-\cos \left(\frac{(i+1 / 3) \pi}{n}\right) \geqq\left(\frac{2 \pi}{3 n}\right) \min \left\{\sin \left(\frac{(i \pm 1 / 3) \pi}{n}\right)\right\} \geqq\left(\frac{\pi}{3 n}\right) \sin \theta_{i},
$$

since $n \geqq 2$ and $\sin \theta_{i} \leqq \min \left\{\sin \left(\left(i \pm \frac{1}{3}\right) \pi / n\right)\right\}$ unless $i=(n+1) / 2$, in which case $\sin \theta_{i} \leqq$ $2 \min \left\{\sin \left(\left(i+\frac{1}{3}\right) \pi / n\right)\right\}$. Therefore

$$
c_{i}^{-1}=\int_{-1}^{1}\left[\frac{T_{n}(x)}{x-t_{i}}\right]^{2 r} d x \geqq\left(\left(\frac{5 \pi}{n}\right) \sin \theta_{i}\right)^{-2 r}\left(\frac{\pi}{3 n}\right) \sin \theta_{i} .
$$

Thus

$$
c_{i} \leqq 15\left(\frac{5 \pi \sin \theta_{i}}{n}\right)^{2 r-1}=15\left(\frac{5 \pi \sqrt{1-t_{i}^{2}}}{n}\right)^{2 r-1} \leqq C_{r}\left(\frac{\sqrt{1-t_{i}^{2}}}{N}\right)^{2 r-1}
$$

To complete the estimate (3.7), observe that

$$
\left|H_{t_{i}}-\lambda_{t_{i}}\right|(x)=c_{i} \begin{cases}\int_{-1}^{x} \delta_{t_{i}}(y) d y, & x \leqq t_{i}, \\ \int_{x}^{1} \delta_{t_{i}}(y) d y, & x>t_{i} .\end{cases}
$$


Therefore,

$$
\left|H_{t_{i}}-\lambda_{t_{i}}\right|(x) \leqq c_{i} \int_{\left|x-t_{i}\right|}^{2} y^{-2 r} d y \leqq \frac{c_{i}\left|x-t_{i}\right|^{-2 r+1}}{(2 r-1)} .
$$

Combined with (3.12), this proves (3.7) for $t=t_{i}$. Hence (3.6) is now verified for $t=t_{i}$.

For the general case $t_{i+1}<t<t_{i}$, define $\lambda_{t}:=\lambda_{t_{i}}$. Note that, since $\sin \theta / \theta$ is decreasing on $[0, \pi]$ (cf. 2.5),

$$
\begin{aligned}
\frac{\pi \sqrt{1-t^{2}}}{3 n} \leqq \pi \min \left\{\sin \theta_{i}, \sin \theta_{i+1}\right\} / n & \leqq \int_{\theta_{i}}^{\theta_{i+1}} \sin \theta d \theta=t_{i}-t_{i+1} \\
& \leqq 3 \pi \min \left\{\sin \theta_{i}, \sin \theta_{i+1}\right\} / n \leqq \frac{3 \pi \sqrt{1-t^{2}}}{n}
\end{aligned}
$$

Therefore,

$$
\begin{aligned}
\int_{-1}^{1}\left|H_{t}-\lambda_{t}\right|(x)|x-t|^{s-1} w(x) d x & =\int_{-1}^{1}\left|H_{t}-\lambda_{t_{i}}\right|(x)|x-t|^{s-1} w(x) d x \\
\leqq & \int_{-1}^{1}\left|H_{t}-H_{t_{i}}\right|(x)|x-t|^{s-1} w(x) d x \\
& +\int_{-1}^{1}\left|H_{t_{i}}-\lambda_{t_{i}}\right|(x)|x-t|^{s-1} w(x) d x
\end{aligned}
$$

This first term, via (A2), is bounded by

$$
\begin{aligned}
\int_{t}^{t_{i}}|x-t|^{s-1} w(x) d x & \leqq A_{2}\left(t_{i}-t\right)^{s} w(t) \leqq A_{2}\left(t_{i}-t_{i+1}\right)^{s} w(t) \\
& \leqq A_{2}\left(\frac{3 \pi \sqrt{1-t^{2}}}{n}\right)^{s} w(t) \leqq C_{r, s}\left(\frac{\sqrt{1-t^{2}}}{N}\right)^{s} w(t)
\end{aligned}
$$

(The application of Assumption (A2) is valid provided, e.g., $\min \left\{1-t_{i}^{2}, 1-t_{i+1}^{2}\right\} \geqq$ $\gamma\left(t_{i}-t_{i+1}\right)$. The reader may easily check that this holds if the constant $\kappa$, mentioned in the corollary and the subsequent discussion, is chosen sufficiently large, depending on $r$ and $\gamma$. This observation applies as well to the application of Assumptions (A2) and (A3) in the next set of inequalities.) Since (3.7)-(3.8) hold for $t_{i}$, the second term is bounded by $\left(\delta:=\sqrt{1-t^{2}} / N\right)$

$$
\begin{aligned}
C_{r} \delta^{2 r-1} & \int_{|x-t| \geqq 2\left|t_{i}-t_{i+1}\right|}\left|x-t_{i}\right|^{-2 r+1}|x-t|^{s-1} w(x) d x+\int_{|x-t| \leqq 2\left|t_{i}-t_{i+1}\right|}|x-t|^{s-1} w(x) d x \\
& \leqq C_{r}(2 \delta)^{2 r-1} \int_{|x-t| \geqq 2\left|t_{i}-t_{i+1}\right|}|x-t|^{s-2 r} w(x) d x+2^{s} A_{2}\left|t_{i}-t_{i+1}\right|^{s} w(t) \\
& \leqq C_{r}(2 \delta)^{2 r-1} A_{3}\left(2\left|t_{i}-t_{i+1}\right|\right)^{s-2 r+1} w(t)+2^{s} A_{2}\left|t_{i}-t_{i+1}\right|^{s} w(t) \\
& \leqq \tilde{C}_{r, s}\left(\frac{\sqrt{1-t^{2}}}{N}\right)^{s} w(t),
\end{aligned}
$$

assuming (A2) and (A3) hold. Therefore, (3.6) is now proved for all $t \in[-1,1]$, and hence the estimate (3.1) is established for smooth $u$ and for weights $w$ satisfying Assumptions (A1)-(A3). In fact, in view of the lemma, one can improve (3.6), and hence $(3.1)$, by replacing the expression $\left(\sqrt{1-t^{2}} / N\right)^{s}$ by $\min \left\{\left(\sqrt{1-t^{2}} / N\right)^{s},\left(1-t^{2}\right)^{s}\right\}$. 
To extend the result to more general $u$, define, for positive integers $s$,

$$
\|u\|_{w, s}=\int_{-1}^{1}\left[|u(x)|+\left|u^{(s)}(x)\right|\left(1-x^{2}\right)^{s}\right] w(x) d x
$$

where $u^{(s)}$ is interpreted as a weak derivative. Define

$$
Y_{w}^{s}:=\left\{u \in L_{\text {loc }}^{1}(]-1,1[):\|u\|_{w, s}<\infty\right\} .
$$

Assuming (A2)-(A3), we have $w(t)>0$ for $|t|<1$ (provided $w \neq 0$ ). Then $Y_{w}^{s}$ is a Banach space having $C^{\infty}([-1,1])$ as a dense subspace ${ }^{1}$. Using this density, we arrive at the following theorem, which summarizes our results.

THEOREM 2. Let $w$ be a positive, integrable function on $[-1,1]$ satisfying Assumptions (A1)-(A3), where $s$ in Assumption (A2) is some positive integer. Let $u \in Y_{w}^{s}$ (see (3.13)-(3.14) for the definition). Then for any positive integer $N \geqq s-1$,

$$
\inf _{P \in \mathscr{P}_{N}} \int_{-1}^{1}|u-P|(x) w(x) d x \leqq C_{s, w} \int_{-1}^{1}\left|u^{(s)}(x)\right| \min \left\{\left(\frac{\sqrt{1-x^{2}}}{N}\right)^{s},\left(1-x^{2}\right)^{s}\right\} w(x) d x
$$

where $\mathscr{P}_{N}$ denotes polynomials of degree not exceeding $N$ and $C_{s, w}$ is a constant independent of $N$ and $u$.

Remark 1. If $u^{(s)}(x)\left(1-x^{2}\right)^{s / 2}$ is integrable on $[-1,1]$, the above estimate may be simplified to yield

$$
\inf _{P \in \mathscr{P}_{N}} \int_{-1}^{1}|u-P|(x) w(x) d x \leqq C_{s, w} N^{-s} \int_{-1}^{1}\left|u^{(s)}(x)\right|\left(1-x^{2}\right)^{s / 2} w(x) d x .
$$

Remark 2. Assumptions (A2)-(A3) imply, in particular, that $w(t)>0$ for $|t|<1$, unless $w \equiv 0$. But such a condition is necessary for an estimate such as (3.15) to hold. To see this, suppose that $w$ is continuous at $t$ and $w(t)=0$. Choose a sequence $\left\{u_{j}\right\}$ of smooth functions with $u_{j}^{(s)}$ positive, supported in $\{x+t:|x| \leqq 1 / j\}$ and $\int_{-1}^{1} u_{j}^{(s)}(x) d x=$ 1 (take $j \geqq 1 /(1-|t|)$ for simplicity). Then $u_{j}$ converges in $L^{1}$ to $u(x):=$ $H_{t}(x)(x-t)^{s-1} /(s-1)$ ! as $j$ tends to infinity. The right-hand side of (3.15) applied to $u_{j}$ tends to zero. However, the left-hand side certainly will not do so since $u \notin \mathscr{P}_{N}$ for any $N$.

4. Higher-order error estimates for Gaussian quadrature. In this section, we combine the results of previous sections to prove an estimate of the form (2.9). From (2.8), we know that

$$
\left|e_{N}(f)\right| \leqq C \inf _{P \in \mathscr{P}_{2 N-2}} \int_{-1}^{1}\left|f^{\prime}-P\right|(x) w(x) d x,
$$

where $w(x):=\min \left\{\sqrt{1-x^{2}} / N, 1-x^{2}\right\}$. It is easy to check that, if a collection of weights $w_{i}$ satisfies (A1), (A2) or (A3), then so does the weight function $\min \left\{w_{i}\right\}$. Thus

${ }^{1}$ Proof. Define $u_{r}(x):=u(r x), 0<r<1$. Then as $r \rightarrow 1, u_{r} \rightarrow u$ in $Y_{w}^{s}$. Let $u_{r, \varepsilon}$ be obtained from $u_{r}$ by mollifying: $u_{r, \varepsilon}:=u_{r} * \delta_{\varepsilon}$ where supp $\left\{\delta_{\varepsilon}\right\}=[-\varepsilon, \varepsilon]$ and $\delta_{\varepsilon}$ has integral one and is smooth. Then $u_{r, \varepsilon}$ is smooth on $[-1,1]$ for $\varepsilon \leqq 1-r$, and $u_{r, \varepsilon} \rightarrow u_{r}$ in $Y_{w}^{s}$. Now let $r \rightarrow 1, \varepsilon:=1-r \rightarrow 0$ to get $u_{r, \varepsilon} \rightarrow u$ in $Y_{w}^{s}$. 
Theorem 2 applies to the weight $w$ since it is a minimum of two Jacobi weights, yielding

$$
\begin{aligned}
\left|e_{N}(f)\right| & \leqq C_{s} \int_{-1}^{1}\left|f^{(s)}(x)\right| \min \left\{\left(\frac{\sqrt{1-x^{2}}}{N}\right)^{s-1},\left(1-x^{2}\right)^{s-1}\right\} w(x) d x \\
& =C_{s} \int_{-1}^{1}\left|f^{(s)}(x)\right| \min \left\{\left(\frac{\sqrt{1-x^{2}}}{N}\right)^{s},\left(1-x^{2}\right)^{s}\right\} d x .
\end{aligned}
$$

We summarize this final result as

THEOREM 3. Let $e_{N}(f)$ denote the error in N-point Gaussian quadrature approximation to the integral of $f$ on $[-1,1]$ (see (2.1)-(2.2) for definitions). Suppose that $\left(1-x^{2}\right)^{s} f^{(s)}(x)$ (weak derivative) is integrable on $[-1,1]$, i.e., $f \in Y_{1}^{s}$ where $s$ is any integer such that $1 \leqq s \leqq 2 N$. Then

$$
\left|e_{N}(f)\right| \leqq C_{s} \int_{-1}^{1}\left|f^{(s)}(x)\right| \min \left\{\left(\frac{\sqrt{1-x^{2}}}{N}\right)^{s},\left(1-x^{2}\right)^{s}\right\} d x,
$$

where $C_{s}$ is independent of $N$ and $f$.

Remark 3. If $f^{(s)}(x)\left(1-x^{2}\right)^{s / 2}$ is integrable on $[-1,1]$, the above estimate simplifies to

$$
\left|e_{N}(f)\right| \leqq C_{s} N^{-s} \int_{-1}^{1}\left|f^{(s)}(x)\right|\left(1-x^{2}\right)^{s / 2} d x
$$

This is the estimate anticipated in (1.1).

Remark 4. Suppose $f(x)=(1-x)^{\sigma} g(x)$, where $\sigma>-1$ and $g \in C^{s}([-1,1])$, where $s$ is the least integer greater than $2 \sigma+2$. Then applying Theorem 3 to $f$ yields, for any $\varepsilon>0$,

$$
\begin{aligned}
\left|e_{N}(f)\right| & \leqq C\left\{\int_{-1}^{1-\varepsilon}(1-x)^{\sigma-s}\left(1-x^{2}\right)^{s / 2} N^{-s} d x+\int_{1-\varepsilon}^{1}(1-x)^{\sigma-s}\left(1-x^{2}\right)^{s} d s\right\} \\
& \leqq C\left\{N^{-s} \varepsilon^{\sigma-s / 2+1}+\varepsilon^{\sigma+1}\right\} .
\end{aligned}
$$

Taking $\varepsilon=N^{-2}$ yields the bound

$$
\left|e_{N}(f)\right| \leqq C N^{-2 \sigma-2}
$$

in agreement with the asymptotic extimate of Davis and Rabinowitz [1, (4.6.1.13)].

Remark 5 . The weight $\sqrt{1-x^{2}}$ in the error bounds above arises primarily because the Gaussian quadrature points form a quadratically graded mesh near the boundary points $x= \pm 1$; cf. (2.4). Similar bounds can thus be obtained for composite rules on such a mesh. To be precise, let $y_{i}=-1+(i / n)^{2}$ for $i=0,1, \cdots, n$, and suppose that $\left\{w_{j}, \xi_{j}: j=1, \cdots, J\right\}$ is a fixed quadrature rule of order $s$ on $[0,1]$; i.e., suppose there is some function $K \in L^{\infty}([0,1])$ such that for all sufficiently smooth $f$

$$
\int_{0}^{1} f(\xi) d \xi-\sum_{j=1}^{J} w_{j} f\left(\xi_{j}\right)=\int_{0}^{1} f^{(s)}(\xi) K(\xi) d \xi
$$

with $s$ some positive integer (we assume $\xi_{j} \in[0,1]$ for all $j$ ). Define, for $i=1, \cdots, n$,

$$
I_{i}(f)=\Delta y_{i} \sum_{j=1}^{J} w_{j} f\left(y_{i-1}+\xi_{j} \Delta y_{i}\right)
$$

where $\Delta y_{i}=y_{i}-y_{i-1}$. For $i=n+1, \cdots, 2 n$, define $I_{i}(f)$ by reflection:

$$
I_{n+i}(f)=I_{n+1-i}(g), \quad g(x)=f(-x) \text { for } x \in[-1,0] .
$$


Then by simple scaling we have

$$
\left|\int_{y_{i-1}}^{y_{i}} f(x) d x-I_{i}(f)\right| \leqq\|K\|_{L^{\infty}([0,1])}\left(\Delta y_{i}\right)^{s} \int_{y_{i-1}}^{y_{i}}\left|f^{(s)}(x)\right| d x
$$

Using the fact that, for $2 \leqq i \leqq n$,

$$
\Delta y_{i} \leqq \frac{3(i-1)}{n^{2}} \leqq 3 \min \left\{\frac{\sqrt{1-y_{i-1}^{2}}}{n}, 1-y_{i-1}^{2}\right\}
$$

together with the symmetry of the quadrature rules around $x=0$ thus yields

$$
\left|\int_{y_{1}}^{-y_{1}} f(x) d x-\sum_{i=2}^{2 n-1} I_{i}(f)\right| \leqq 3\|K\|_{L^{\infty}} \int_{y_{1}}^{-y_{1}}\left|f^{(s)}(x)\right| \min \left\{n^{-s}\left(1-x^{2}\right)^{s / 2},\left(1-x^{2}\right)^{s}\right\} d x
$$

Provided that the end intervals can be estimated in a similar way, one has a result as in Theorem 3. Specifically, if $\xi_{j} \neq 0$ for all $j$ (i.e., if $\xi=0$ is not a quadrature point), then

$$
|K(\xi)| \leqq C \xi^{s}, \quad \xi \in[0,1]
$$

and we easily see that

$$
\left|\int_{-1}^{y_{1}} f(x) d x-I_{1}(f)\right| \leqq C \int_{-1}^{y_{1}}\left|f^{(s)}(x)\right|(1+x)^{s} d x .
$$

Using symmetry again and the definition of $y_{1}$, we thus find

$$
\left|\int_{-1}^{1} f(x) d x-\sum_{i=1}^{2 n} I_{i}(f)\right| \leqq C \int_{-1}^{1}\left|f^{(s)}(x)\right| \min \left\{n^{-s}\left(1-x^{2}\right)^{s / 2},\left(1-x^{2}\right)^{s}\right\} d x
$$

for some constant $C$ independent of $f$ and $n$. Of course, if $\xi=0$ is a quadrature point, a result such as (4.2) cannot hold since the right-hand side is finite for, say, $f(x)=(1+x)^{-1 / 2}$.

Acknowledgments. We wish to thank Professor G. P. Nevai for informing us of the work of Ky [2]. Professor Nevai has also developed an alternate (related) method to that in $\$ 3$ for proving weighted approximation estimates.

\section{REFERENCES}

[1] P. J. DAVIS ANDP. Rabinowitz, Methods of Numerical Integration, Academic Press, New York, 1975.

[2] N. X. KY, On Jackson and Bernstein type approximation theorems in the case of approximation by algebraic polynomials in $\mathscr{L}_{p}$-spaces, Studia Sci. Math. Hungar., 9 (1974), pp. 405-415.

[3] J. PITKÄRANTA AND L. R. SCOTT, Error estimates for the combined spatial and angular approximations of the transport equation for slab geometry, this Journal, 20 (1983), pp. 922-950.

[4] G. SzEgö, Orthogonal Polynomials, 4th ed., AMS Colloquium Publications, 23, American Mathematical Society, Providence, RI, 1975.

[5] A. F. TimaN, Theory of Approximation of Functions of a Real Variable, Pergamon Press, Oxford, 1963. 